\title{
ÍNDICE MULTICRITÉRIO DE BEM ESTAR SOCIAL RURAL EM UM MUNICÍPIO DA REGIÃO AMAZÔNICA
}

\author{
Eliane Gonçalves Gomes * \\ Embrapa Sede - SGE \\ Brasília - DF \\ eliane.gomes@embrapa.br
}

João Carlos Correia Baptista Soares de Mello

Departamento de Engenharia de Produção

Universidade Federal Fluminense (UFF)

Niterói - RJ

jcsmello@producao.uff.br

João Alfredo de Carvalho Mangabeira

Embrapa Monitoramento por Satélite

Campinas - SP

manga@cnpm.embrapa.br

* Corresponding author / autor para quem as correspondências devem ser encaminhadas

Recebido em 11/2006; aceito em 01/2008 após 1 revisão

Received November 2006; accepted January 2008 after one revision

\begin{abstract}
Resumo
Neste artigo propôs-se a construção de um índice multicritério para avaliar o bem estar social de um grupo de agricultores. Abreviadamente, o índice foi designado de "bem estar rural". Para a construção do índice foram considerados aspectos relacionados às condições de habitação, saúde, trabalho e infraestrutura. $\mathrm{Na}$ determinação dos pesos para a construção do índice, usou-se tanto a abordagem MACBETH clássica, quanto uma variante otimista, que leva em conta os pesos mais favoráveis dentro do intervalo definido pelo MACBETH. Esta atitude é motivada por uma premissa de otimismo. Esta justifica-se pelo fato de os pesquisadores de campo terem constatado que os agricultores valorizavam mais aquilo que possuíam em detrimento do que não tinham. Os resultados da aplicação desse modelo indicaram uma tendência de crescimento médio do bem estar rural, no grupo avaliado.
\end{abstract}

Palavras-chave: multicritério; avaliação otimista; MACBETH.

\begin{abstract}
In this paper we proposed a multicriteria index to evaluate rural social welfare. This index was named "rural welfare". We took into account some criteria related to health, work, infrastructure and type of habitation. We used the classical MACBETH approach and an optimistic variation to determine the weights. The last one uses the most favorable weights (for each farmer) belonging to an interval defined by the MACBETH, instead of the MACBETH weights themselves. This approach was motivated by an optimist behavior assumption. This premise is justified as the field researchers noticed that the rural producers overvalue their belongings and under-evaluate things that they do not have. The results showed that the rural welfare has, in average, growth tendency for the group of family farmers under evaluation.
\end{abstract}

Keywords: multicriteria; optimistic evaluation; MACBETH. 


\section{Introdução}

O conceito de desenvolvimento de uma região não deve considerar somente aspectos relativos ao crescimento econômico e ao aumento da renda (seja nacional ou per capita). Sem negligenciá-los, é necessário levar em contar também fatores não traduzíveis em variáveis econômicas, alguns de avaliação bastante subjetiva (Kageyama \& Rehder, 1993).

Há na literatura dois enfoques principais para avaliar o bem estar rural. O primeiro considera somente o critério econômico (geralmente, renda). Destacam-se, por exemplo, os trabalhos de Barros et al. (1997), Mariano \& Lima (1998), Corrêa et al. (2003). A segunda vertente engloba outros aspectos além do econômico: aqueles relativos às condições de habitação, educação, saúde, trabalho etc. Neste enfoque encontram-se as pesquisas de Kageyama \& Rehder (1993), Mapurunga (2000), Khan et al. (2001), entre outros.

Neste artigo propõe-se a construção de um índice relacionado ao bem estar social rural semelhante ao do segundo enfoque. Dado que este usa múltiplos fatores, alguns de elevada subjetividade, é recomendável o uso de métodos multicritério (aspecto não abordado na literatura consultada). Foram consideradas algumas características relacionadas às condições de habitação, saúde, trabalho e infra-estrutura. Alguns destes têm relação direta com a renda. Entretanto, em oposição às abordagens usuais, o critério econômico não é explícito no modelo aqui proposto, de forma similar à abordagem de Sousa et al. (2005) para medir qualidade de vida.

O estudo foi realizado com base em questionários de campo aplicados a um grupo de produtores rurais do município de Machadinho d'Oeste (estado de Rondônia, Brasil). Foram considerados no estudo somente os produtores amostrados nas pesquisas de campo e que se mantiveram na região ao longo de quatro períodos de tempo $(1989,1996,1999,2002)$. O objetivo é avaliar, com base no índice proposto, a evolução do bem estar social desses produtores.

$\mathrm{Na}$ impossibilidade de obter as opiniões individuais de cada agricultor (o que ainda obrigaria a modelagem de um problema multidecisor), considerou-se como decisor um pesquisador que estuda o perfil dos agricultores e da agricultura da região desde a década de 1980. Este decisor participou da escolha dos critérios e emitiu os juízos de valor inter e intracritério. Os juízos de valor foram transformados em escalas cardinais pelo método MACBETH (Bana e Costa et al., 2005). O uso do método MACBETH em uma outra aplicação na área agrícola pode ser vista, por exemplo, em Ensslin et al. (1998).

Dada uma constatação de otimismo dos agricultores e a dificuldade do especialista em fazer uma análise fina, os pesos foram calculados segundo um modelo de otimização.

Os resultados sugerem uma evolução positiva do índice proposto, indício de que as práticas agrícolas da região são propulsoras de bem estar social rural.

\section{Localização e caracterização da área de estudo}

Machadinho d'Oeste é um município do estado de Rondônia, localizado entre Ariquemes e Jaru, entre as coordenadas geográficas $61^{\circ} 47^{\prime}$ e $63^{\circ} 00^{\prime}$ de longitude WGr e $9^{\circ} 19^{\prime}$ e $10^{\circ} 00^{\prime}$ de latitude S, distanciado aproximadamente $400 \mathrm{~km}$ da capital Porto Velho (Figura 1). 


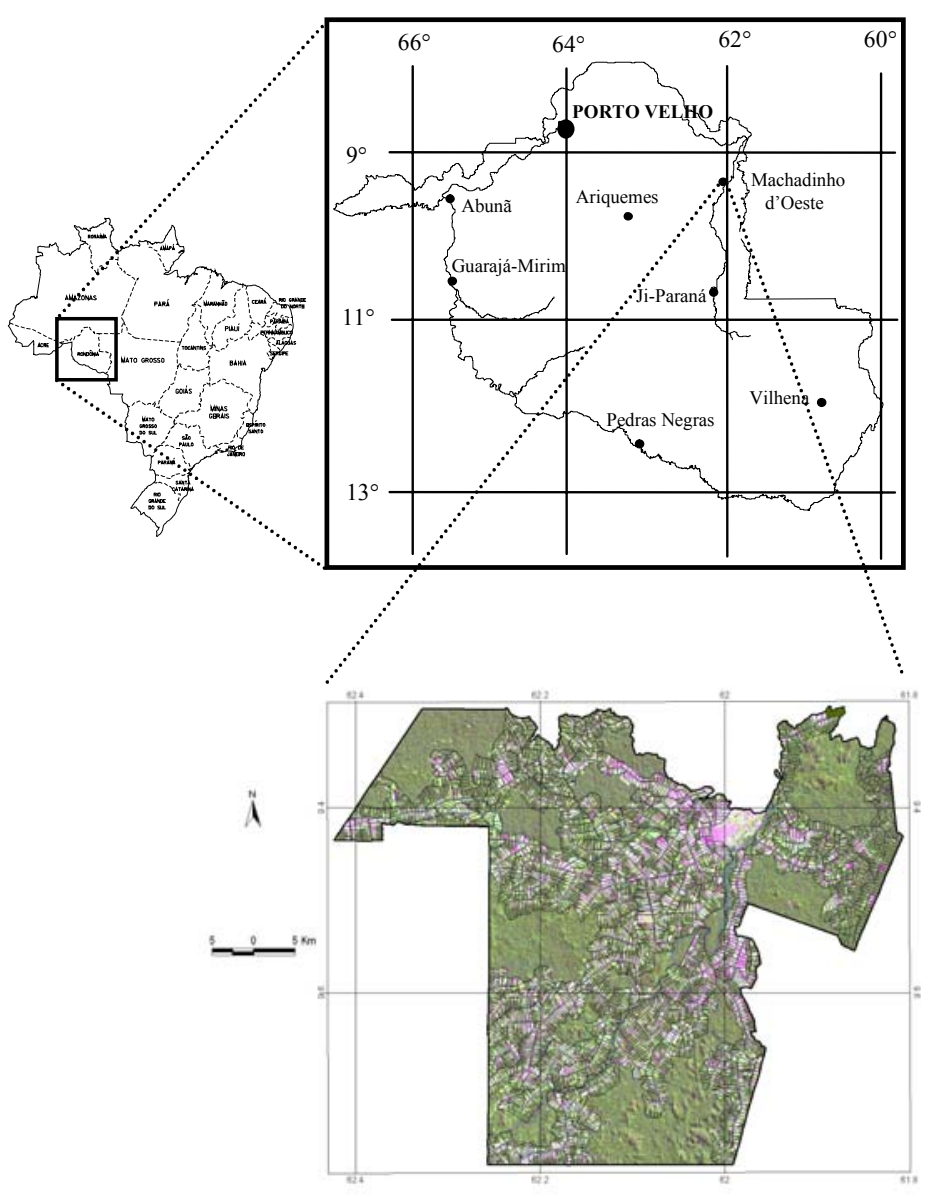

Figura 1 - Localização de Machadinho d'Oeste (Miranda et al., 2002b).

Antes de ser elevado à condição de município em 1988, Machadinho d'Oeste surgiu como um projeto de assentamento (PA) rural. Enquanto a maioria dos assentamentos rurais de Rondônia foi implantada seguindo o modelo "espinha de peixe", no PA de Machadinho as estradas e lotes acompanharam a topografia. Além disso, o PA também incluiu reservas florestais, posteriormente decretadas como Reservas Extrativistas Estaduais. Ao ser instituído como município, seus limites foram ampliados e novas áreas foram incorporadas (4 outros projetos de colonização e 8 centros urbanos), em um total de $8.556 \mathrm{~km}^{2}$.

O PA Machadinho foi dimensionado inicialmente para um total de 3.000 famílias de colonos, das quais mais de 2.000 já haviam chegado em 1984. Nesse ano, esse PA já tinha infraestrutura mínima para a colonização agrícola, como estradas, núcleos urbanos de apoio, projeto fundiário implantado, lotes demarcados etc.

Em 1986 foi definido um projeto de pesquisa para acompanhar os sistemas de produção agrícola praticados por pequenos agricultores desta região. Pesquisas de campo são feitas a cada três anos, com aplicação de questionários, com cerca de 250 variáveis agrosocioeconômicas e ambientais (descritores de localização e situação das propriedades - 
12 variáveis, descritores socioeconômicos - 83 variáveis, descritores agronômicos - 30 variáveis para cada cultura e 14 variáveis para a pecuária), sobre uma amostra de propriedades. A ficha de levantamento da propriedade rural tenta descrever, de forma mais objetiva quanto possível, a realidade dos agricultores. Foi testada e elaborada em função das informações disponíveis sobre o objeto em estudo e dos objetivos do trabalho.

A cada ano, o uso das terras é monitorado por imagens de satélites e espacializado em um Sistema de Informações Geográficas (SIG). A partir da implementação do assentamento no início dos anos de 1980, a paisagem original tem sido transformada pelos colonos em um mosaico composto por remanescentes florestais, vegetação secundária, pastagens, culturas agrícolas e pequenas áreas urbanizadas. Em Batistella et al. (2003, 2000) e Batistella (2001) encontram-se estudos sobre as mudanças da paisagem e do uso e cobertura das terras em Machadinho d'Oeste.

Em 1986, primeiro ano da pesquisa, foi realizado um esforço de amostragem que cobriu cerca de $15 \%$ dos lotes (uma primeira amostra de 588 lotes, $20 \%$ do total, estava com muitos desocupados e foi redimensionada): 125 na Gleba 1, 228 na Gleba 2, 47 na Gleba 3, 38 na Gleba 6, representativo de 54\% (438) do total de lotes ocupados. Essa amostra aleatória e estratificada foi geocodificada em um SIG.

Os resultados do primeiro levantamento de dados in loco permitiram definir um primeiro perfil dos agricultores recém chegados e de sua agricultura. Em 1989, um novo levantamento permitiu elaborar e espacializar em SIG o perfil da agricultura e dos agricultores. Em 1993, outra etapa foi realizada, aprofundando aspectos vinculados à economia e ao meio ambiente.

A pesquisa de campo de 1996 foi feita nas mesmas 438 propriedades investigadas em 1986. Porém, houve visitação às propriedades não ocupadas em 1986, daquela amostra sorteada inicialmente de 588 propriedades. O primeiro tratamento dos dados mostrou que, em 10 anos, houve mudanças profundas nas propriedades, tanto na estrutura quanto nos sistemas de produção (Miranda et al., 1997).

Em um novo levantamento de campo em 1999 foram entrevistados 438 agricultores, cujos sistemas de produção foram caracterizados. Caso a propriedade tivesse mudado de proprietário, o novo responsável era entrevistado e, dessa forma, foi elaborado um novo perfil dos agricultores e da agricultura (Miranda et al., 2002a).

Em setembro e outubro de 2002, conforme apresentado em Mangabeira et al. (2005), um novo levantamento foi conduzido junto às propriedades estudadas em anos anteriores, para atualização das variáveis sobre os sistemas de produção praticados. Nessa ocasião, foram entrevistados 327 proprietários. As publicações referentes a esses 20 anos de pesquisa em Machadinho d'Oeste podem ser encontradas em Miranda (2005a, 2005b).

\section{Apoio multicritério à decisão}

O Apoio Multicritério à Decisão (AMD) consiste em um conjunto de métodos e técnicas para auxiliar ou apoiar pessoas e organizações a tomarem decisões, quando da presença de uma multiplicidade de critérios (Gomes et al., 2002).

No AMD buscam-se construir modelos que lidam com os juízos de valor subjetivos, ou seja, pressupõe aceitar que a subjetividade é um elemento desejável e estará presente em todo o processo de decisão. Dessa forma, a estrutura de valores dos decisores é associada aos critérios existentes e que serão usados na avaliação das alternativas (Yu, 1985). 
A análise de decisão com múltiplos critérios é um processo interativo, e pode ser apresentado como a seqüência das seguintes etapas (Gomes et al., 2004): 1) Identificação dos decisores e seus objetivos; 2) Definição das alternativas; 3) Definição dos critérios relevantes para o problema de decisão; 4) Avaliação das alternativas em relação aos critérios; 5) Determinação da importância relativa dos critérios; 6) Avaliação global de cada alternativa; 7) Análise de sensibilidade; 8) Recomendação de cursos de ação; 9) Implementação. Uma outra etapa importante é a escolha do método a ser usado, que deve depender mais da sua adequação à estrutura de preferências dos decisores, do que da preferência do analista por determinados modelos e métodos.

Em relação à etapa 3, cabe ressaltar que em um problema de decisão complexo, os critérios podem ser estruturados na forma de hierarquia ou árvore, na qual o nível do critério mais elevado é decomposto em níveis mais detalhados (Gomes et al., 2004).

Deve-se ainda atentar que uma família de critérios, ou seja, o conjunto de critérios empregados em uma determinada situação de decisão, deve satisfazer a três condições ("axiomas de Roy") para que seja uma família coerente de critérios (Roy \& Bouyssou, 1993): Exaustividade; Coesão; Não Redundância.

Em AMD, os juízos de valor dos decisores são expressos por meio de suas estruturas de preferência entre pares de alternativas ou critérios. As quatro relações fundamentais são indiferença, preferência estrita, preferência fraca e incomparabilidade (Roy \& Bouyssou, 1993; Gomes et al., 2002, 2004).

\section{Modelagem do problema}

\subsection{Decisor e critérios}

A primeira fase de uma modelagem multicritério é a fase de estruturação, composta de três etapas. Na primeira etapa foi identificado o decisor. Na impossibilidade de obter as opiniões individuais de cada agricultor, atuou como decisor um pesquisador que participou de todas as campanhas de campo em Machadinho d'Oeste, e que estuda o perfil dos agricultores e da agricultura da região desde 1986. Este pesquisador, durante todos trabalhos de campo, participou ativamente das entrevistas, conheceu pessoalmente diversos produtores, as suas opiniões e aspirações, especialmente durante diversos diagnósticos participativos. O decisor participou da escolha dos critérios e emitiu os juízos de valor inter e intracritério.

$\mathrm{Na}$ avaliação intercritério houve certa dificuldade na emissão dos julgamentos, já que, na opinião do decisor, os diversos agricultores valorizam de forma diferente os critérios. Têm tendência a valorizar o que possuem, o que se caracteriza como uma atitude otimista. Este fato sugere a escolha de um modelo de pesos variáveis, e foi determinante na escolha do método multicritério usado, como visto adiante. Apesar de haver variações em julgamentos cardinais, as preferências ordinais sobre os critérios foram observadas como sendo razoavelmente invariantes.

A escolha dos critérios de bem estar social rural ficou restrita às variáveis do questionário usado nos levantamentos de campo em Machadinho d'Oeste. Essas variáveis, conforme previamente mencionado, são descritores de localização e situação das propriedades, socioeconômicos e agronômicos. No primeiro grupo estão as variáveis: gleba, lote, nome do agricultor, idade, local de origem, tempo na região, áreas (total, cultivada, de mata, de pastagens etc.), entre outras. No grupo dos descritores socioeconômicos encontram-se 
assistência técnica, uso do núcleo urbano de apoio rural, condição fundiária, participação em grupos comunitários, cooperativas, uso de financiamentos, total de dias parados, número de pessoas e de ativos na família, tempo de dedicação ao lote, tipo de instalações e equipamentos, principais problemas e necessidades etc. As variáveis agronômicas investigadas, por tipo de cultura, são área plantada, produção, técnicas de tratamento e conservação do solo, insumos, entre outras.

Dentre essas variáveis, a seleção ficou condicionada aos aspectos socioeconômicos e de localização, mais especificamente aos critérios referentes às condições de habitação, saúde, trabalho e infra-estrutura. Alguns desses aspectos têm relação direta com a renda, mas o critério econômico não é explícito no modelo proposto, já que esta variável não consta do questionário de campo. Os critérios eleitos foram: participação em NUAR (Núcleos Urbanos de Apoio Rural); participação em cooperativas; membro de sindicato; membro de associação; percentual de dias ativos; participação em grupo não sindical (comunitário ou religioso); se é proprietário do lote; característica das instalações (poço, casa - alvenaria, madeira, pau roliço, lona -, energia).

A escolha dos critérios baseou-se na seguinte questão: o que poderia motivar ou lograr os agricultores a uma melhor qualidade de vida pela aquisição de um lote no assentamento? Isto porque uma grande ambição de todos os agricultores é a aquisição da terra. Para eles, Rondônia era um mito que se espelhava no sonho da posse da terra (o "mito da terra"), no qual o simples acesso à posse da terra garantiria um futuro de progresso (Miranda, 1987). Por outro lado, ao adquirir um lote os produtores teriam que suprir todas suas necessidades, e somente a posse do lote não seria condição suficiente para garantir sua viabilidade social e econômica. A partir do momento em que precisassem de renda extra-agrícola, esse era um sinal da não sustentação familiar via produção na terra. A árvore de critérios resultante é apresenta na Figura 2.

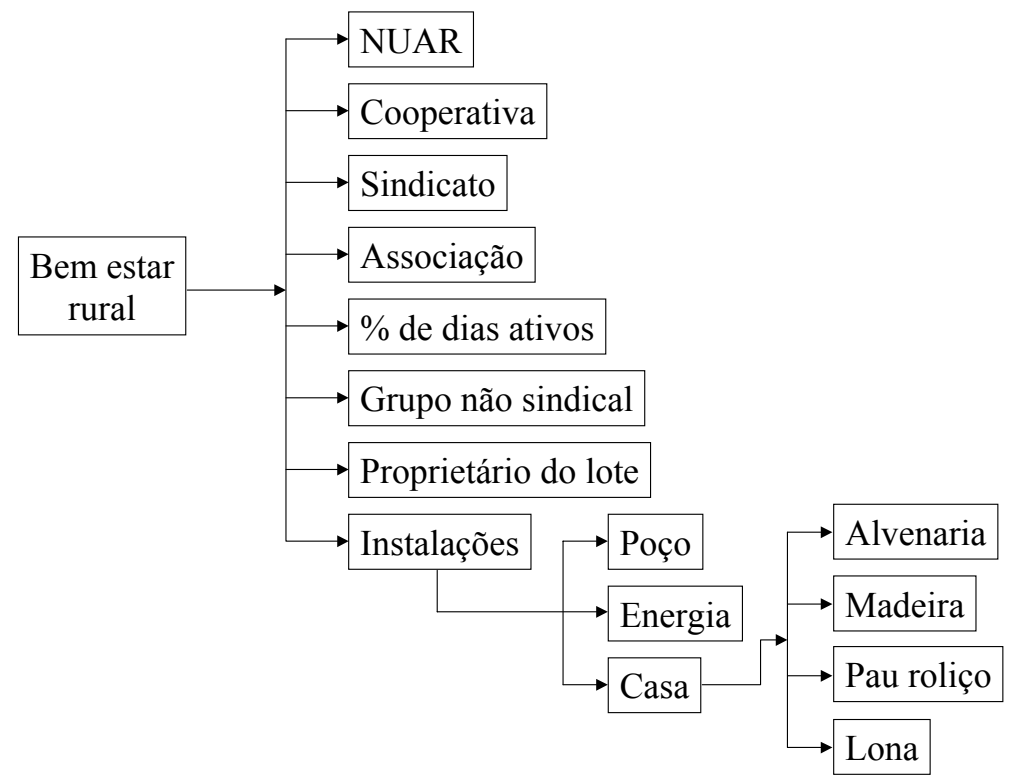

Figura 2 - Árvore de critérios. 


\subsection{Método}

Apesar de a estrutura hierárquica da Figura 2 sugerir, a princípio, o uso do método AHP Analytic Hierarchy Process (Saaty, 1980), este foi preterido pelo fato de permitir inconsistência nos julgamentos do decisor. Um estudo comparativo entre o AHP e outro método aparentemente semelhante, o método MACBETH, pode ser visto em Ensslin et al. (1996). Uma comparação empírica de resultados dos dois métodos pode-se encontrar, por exemplo, em Madeira Júnior (2004).

Bana e Costa \& Vansnick (2008) apresentam uma discussão sobre alguns problemas relacionados ao uso do método AHP. Gomes (2003), Goodwin \& Wright (2004), Thomaz \& Gomes (2006) discutem outros aspectos sobre o uso do AHP. Além da permissão de inconsistências, destacam-se ainda as seguintes desvantagens: conversão da escala verbal para numérica, já que a correspondência entre as duas escalas é baseada em pressupostos não testados; o fato de os pesos serem obtidos sem relação às escalas nas quais os atributos são medidos; interferência de novas alternativas na ordenação gerada (reversão de ranking); grande número de julgamentos pelo agente de decisão; base axiomática não fundamentada, conforme destacado por Dyer (1990).

No presente estudo, o método AHP tem a desvantagem de fornecer um valor único para os pesos, o que inviabilizaria o uso de um modelo de pesos variáveis, anteriormente identificado como mais adequado ao caso em questão.

Um modelo aditivo de pesos variáveis pode ser visto na equação (1), conforme Kao \& Hung (2007), no qual $I_{i}$ é o índice da alternativa $i ; S_{i j}$ é o valor da alternativa $i$ no critério $j$, $j=1 \ldots n ; w_{i j}$ é o peso da alternativa $i$ no critério $j ; l_{j}$ e $u_{j}$ são, respectivamente, os limites inferior e superior para o peso $w_{i j}$. As restrições sobre relações entre os pesos podem ser obtidas das avaliações intercritério do decisor.

$$
\begin{aligned}
& \operatorname{Max} I_{i}=\sum_{j=1}^{n} w_{i j} S_{i j} \\
& \text { sujeito a } \\
& \sum_{j=1}^{n} w_{i j}=1 \\
& l_{j} \leq w_{i j} \leq u_{j}, \forall j
\end{aligned}
$$

O método MACBETH (Bana e Costa \& Vansnick, 1995, 1997, 1999; Bana e Costa et al., 2005) também é, em princípio, um método de pesos fixos. No entanto, ao possibilitar que o decisor escolha um valor dentro de um intervalo, pode ser facilmente adaptado para um modelo de pesos variáveis. Os limites inferior e superior calculados para os pesos fornecem as restrições do modelo (1). Para garantir que o modelo seja fidedigno à opinião do decisor, é necessário incluir restrições que considerem também as relações ordinais entre os pesos dos critérios. Esta inclusão é necessária dado que, no MACBETH, o limite superior para o peso de um critério de menor peso pode ser maior que o limite inferior para o peso de um critério de maior peso. Ressalte-se que, devido às outras restrições, o modelo é cardinal, embora contenha também informação ordinal. O modelo (2), aqui proposto, incorpora essas restrições. 


$$
\begin{aligned}
& \operatorname{Max} I_{i}=\sum_{j=1}^{n} w_{i j} S_{i j} \\
& \text { sujeito a } \\
& \sum_{j=1}^{n} w_{i j}=1 \\
& l_{j} \leq w_{i j} \leq u_{j}, \forall j \\
& w_{i 1} \leq w_{i 2} \\
& \ldots \\
& w_{i n-1} \leq w_{i n}
\end{aligned}
$$

Repare-se que o modelo (2) é extremamente otimista. De um ponto de vista estritamente matemático, deve-se considerar que essa análise otimista é semelhante à análise otimista do método VIP Analysis (Dias \& Clímaco, 2000). Este permite também o uso de avaliações pessimistas, como mostrado em Campos \& Almeida (2006). A opção por uma avaliação otimista vem das informações adquiridas na pesquisa de campo. Constatou-se que os agricultores valorizam mais os itens que possuem, em detrimento dos que não possuem. Há assim uma valorização diferente de cada item, de agricultor para agricultor. Nestas condições, o uso de pesos fixos não representaria adequadamente a opinião de cada agricultor, apenas a opinião do especialista que atuou como decisor, o que seria algo semelhante ao "paradigma do ótimo", criticado por Clímaco (2003). A necessidade de usar pesos diferentes para cada agricultor e a atitude otimista destes justificam o uso do modelo (2).

Destaca-se ainda que, embora originalmente o MACBETH não tenha sido desenvolvido para o emprego de árvores de critérios, não há nada em sua estrutura que impeça este uso.

Isto posto, escolheu-se como método multicritério, a conjunção do método MACBETH com o modelo (2). Devido à dificuldade de operacionalização do método e à pouca influência que teria no resultado final, este modelo foi usado somente para as avaliações intercritério. As avaliações intrasubcritério foram feitas com os resultados do método MACBETH original.

Propostas de uso dos intervalos de pesos fornecidos pelo MACBETH em outros modelos de apoio à decisão baseados em programação linear já foram feitas anteriormente na literatura. Por exemplo, Rangel et al. (2003) usaram os intervalos de pesos obtidos pelo MACBETH para introduzir restrições adicionais ao método UTA de Jacquet-Legreze \& Siskos (1982). Soares de Mello et al. (2002b) propuseram o uso dos intervalos de pesos obtidos pelo MACBETH para impor restrições aos multiplicadores em um modelo DEA. Chaves et al. (2007) usaram o MACBETH para obter os intervalos de pesos a serem usados pelo método Vip Analysis de Dias \& Clímaco (2000).

\subsubsection{MACBETH}

O método MACBETH permite construir uma função de valor intracritério e, por meio de uma análise intercritério, agregar os diversos critérios de avaliação em um critério único de síntese pela atribuição de pesos aos critérios, com respeito às opiniões dos decisores. A seguir é feita uma descrição do método MACBETH, baseada em Bana e Costa \& Vansnick (1995), Corrêa (1996) e Soares de Mello et al. (2002a). 
$\mathrm{Na}$ avaliação intracritério, as preferências são representadas por uma função $v$ e por funções limiares $\mathrm{s}_{k}$, de modo que: $a \mathrm{P}^{(k)} b, \mathrm{~s}_{k}<v(a)-v(b)<\mathrm{s}_{k+1}$. Ou seja, é possível representar numericamente categorias semânticas de diferença de atratividade através de um intervalo de números reais.

A partir da comparação par a par da atratividade das alternativas são atribuídos os pesos aos critérios: dadas duas alternativas, o decisor deve dizer qual a mais atrativa e qual o grau desta atratividade em uma escala semântica $\mathrm{C}_{k}, k=1 . .6$, que tem correspondência com uma escala ordinal: $\mathrm{C}_{1} \equiv$ diferença de atratividade muito fraca $\rightarrow \mathrm{C} 1=\left[\mathrm{s}_{1}, \mathrm{~s}_{2}\right]$ e $\mathrm{s}_{1}=0 ; \mathrm{C}_{2} \equiv$ diferença de atratividade fraca $\left.\rightarrow \mathrm{C} 2=] \mathrm{s}_{2}, \mathrm{~s}_{3}\right] ; \mathrm{C}_{3} \equiv$ diferença de atratividade moderada $\left.\left.\rightarrow \mathrm{C} 3=\right] \mathrm{s}_{3}, \mathrm{~s}_{4}\right]$; $\mathrm{C}_{4} \equiv$ diferença de atratividade forte $\left.\left.\rightarrow \mathrm{C}_{4}=\right] \mathrm{s}_{4}, \mathrm{~s}_{5}\right] ; \mathrm{C}_{5} \equiv$ diferença de atratividade muito forte $\left.\rightarrow \mathrm{C} 5=] \mathrm{s}_{5}, \mathrm{~s}_{6}\right] ; \mathrm{C}_{6} \equiv$ diferença de atratividade extrema $\left.\rightarrow \mathrm{C}_{6}=\right] \mathrm{s}_{6},+[$.

Matematicamente, MACBETH é constituído por quatro problemas de programação linear (PPLs) seqüenciais, que são responsáveis pela análise de consistência cardinal, pela construção da escala de valor cardinal e por revelar as fontes de inconsistência (Bana e Costa \& Vansnick, 1995).

Para operacionalizar o método são construídas matrizes de juízos de valor, as quais facilitam a expressão dos julgamentos absolutos de diferença de atratividade entre os pares de ações. Cada elemento $x_{r s}$ da matriz toma o valor $k$ se o decisor julgar que a diferença de atratividade do par $\left(a_{r}, a_{s}\right)$ pertence à categoria $\mathrm{C}_{k}$. Estes números não têm significado matemático; servem apenas como indicadores semânticos de qual categoria de diferença de atratividade foi atribuída ao par respectivo.

Com este conjunto de julgamentos, o método MACBETH é executado, primeiramente para a verificação de eventuais inconsistências e, posteriormente, para a determinação de uma escala de valor cardinal que represente os julgamentos de valor do decisor. A escala obtida é normalizada e gera os valores dos pesos para as alternativas em avaliação, o que possibilita o uso de um modelo de agregação, em geral, aditivo.

O software que implementa computacionalmente o método faz a análise de coerência dos julgamentos e sugere, em caso de incoerência, como resolvê-la. Por programação linear é sugerida uma escala de valores e os intervalos em que eles podem variar sem tornar o problema inconsistente (problema de programação linear inviável). É ainda facultado ao decisor ajustar graficamente os valores atribuídos, dentro dos intervalos permitidos (análise de sensibilidade). Segundo Bana e Costa \& Vansnick (1997) somente após este ajuste, com a introdução dos conhecimentos dos especialistas, é que fica caracterizada a construção da escala cardinal de valores.

A avaliação intercritério é feita de forma indireta através da comparação de alternativas extremas. O decisor deve construir $n+1$ alternativas fictícias ( $n$ critérios), onde as $n$ primeiras representam a melhor avaliação observada no critério $j$ e a pior observada em todos os outros critérios. A última alternativa fictícia tem as piores avaliações observadas em todos os critérios. A construção da escala cardinal é feita conforme descrição anterior, apenas com a mudança do método de normalização (soma das avaliações deve ser igual a 1). O "peso" para cada critério é dado pela avaliação da alternativa fictícia que apresenta o melhor desempenho nesse critério. 


\section{Resultados e discussão}

\subsection{Descritores de critérios e subcritérios}

O método MACBETH permite construir uma função de valor que faz corresponder a cada alternativa um número que, para o critério em questão, é uma expressão cardinal do valor ou utilidade dessa alternativa para o decisor. O termo utilidade aqui foi usado de forma não técnica, sem recorrer a comparações de loterias, apenas como uma indicação intuitiva.

Deve-se levar em conta que, embora para a maioria dos atributos haja descritores naturais, estes não devem, via de regra, ser usados como função de valor. No entanto, neste artigo, os descritores naturais tinham propriedades especiais, que levaram a que fossem usados.

Para quase todos os critérios e subcritérios o descritor é do tipo binário: tem-se energia ou não se tem, tem-se poço ou não. Assim, a alternativa "ter poço" tem o descritor natural "1" e a alternativa "não ter poço" tem o descritor natural "zero". Devido à normalização adotada pelo MACBETH, estes valores correspondem ao valor de cada uma das alternativas.

Um dos critérios tem um descritor natural não binário: é o percentual de dias ativos. Após várias perguntas ao decisor verificou-se que este considerava que o valor do número de dias ativo era aproximadamente proporcional a esse número. Como a variação desse número de dias era pequena, optou-se por considerar o descritor natural como uma aproximação linear da função de valor. Esta opção evitou a necessidade de um grande número de comparações pareadas, com possibilidades de erro de julgamento por cansaço, e com alteração desprezível no resultado. Deve-se notar que a possibilidade do uso desta aproximação linear decorre da natureza do problema e, principalmente, da atitude do decisor. Para uma discussão, e exemplos, sobre o uso do descritor natural, ou de uma função de valor obtida por comparações pareadas para grupos de decisores diferentes ver Soares de Mello et al. (2004).

Devido à existência de vários critérios com alternativas descritas de forma binária, muitas das "alternativas extremas" (fictícias) usadas na avaliação intercritério são, na verdade, alternativas reais.

A existência de critérios binários tem outra conseqüência importante: o significado dos pesos neste problema multicritério. No método MACBETH, como em qualquer método aditivo, os pesos não representam diretamente a importância de cada critério. Eles estão ligados também às escalas usadas, e o seu quociente representa razões de compensação. Ou seja, quantas unidades o decisor aceita perder (ou ganhar) em um critério como compensação para cada unidade perdida (ou ganha) em outro. No entanto, quando há avaliações binárias esta interpretação deve ser revista. De fato, não se pode falar em perder, por exemplo, $0,7 \mathrm{em}$ um critério em que as alternativas assumem apenas valores 0 ou 1. Neste caso, o peso $p$ para um critério em que a alternativa $\mathrm{A}$ tem valor 1 e a alternativa $\mathrm{B}$ tem valor nulo significa que, para compensar uma avaliação oposta em um critério de peso $q$, deveria haver $q / p$ critérios de peso $p$. Assim, como as escalas são iguais e a avaliação é binária, os pesos refletem realmente a importância de cada critério, numa interpretação semelhante à dos métodos ELECTRE. Para análise de sensibilidade e interpretação dos pesos em métodos ELECTRE ver, por exemplo, Soares de Mello et al. (2005) ou Costa et al. (2007). 


\subsection{Avaliação intrasubcritérios}

$\mathrm{Na}$ avaliação intracritério verifica-se na árvore da Figura 2 que o critério Instalações é decomposto em três subcritérios de nível I, Poço, Energia e Casa. Este, por sua vez, é ainda subdividido nos tipos de casa, Alvenaria, Madeira, Pau roliço e Lona (subcritérios de nível II). Para manter a estrutura hierárquica, a avaliação foi iniciada pelos subcritérios de nível II e, posteriormente, pelos subcritérios de nível I. Todos estes têm importância diferenciada para explicar o bem estar social rural.

$\mathrm{Na}$ execução do método MACBETH deve-se hierarquizar, por ordem decrescente de atratividade, os critérios/subcritérios. Para os subcritérios de nível II e ao considerar que o objetivo é o bem estar social, o decisor propôs a seguinte ordenação: Alvenaria, Madeira, Pau roliço e Lona. Caso o decisor não esteja apto a expressar diretamente essa hierarquia, pode-se proceder a uma fase intermediária de ordenação de preferências.

Esta hierarquia parte do pressuposto de que os produtores dão preferência às casas de alvenaria pelo "status de ser bem sucedido" e pelo conforto. Ao chegar em Machadinho, os acampamentos eram de lona. Posteriormente construíram casas de pau roliço (podiam conseguir esse material no próprio lote), até obter recurso financeiro, a partir da produção, para comprar madeiras serradas para construção de casas de madeira. Como é difícil e caro obter materiais para construção de casas de alvenaria em Machadinho d'Oeste, somente o produtor que consegue bom desempenho em seu sistema de produção, pode construir esse tipo de instalação.

A segunda etapa é fazer uma avaliação intercritério para os subcritérios de nível II, de forma a construir a função de valor para este critério. É construída uma matriz que incorpora os julgamentos. Cada elemento da matriz é preenchido com o julgamento de valor referente à comparação de cada par de critérios. A Figura 3 mostra essa matriz preenchida com os julgamentos para cada comparação pareada entre as alternativas fictícias representantes dos subcritérios de nível II.

\begin{tabular}{|c|c|c|c|c|}
\cline { 2 - 5 } \multicolumn{1}{c|}{} & Alvenaria & Madeira & Pau roliço & Lona \\
\hline Alvenaria & - & Muito forte & Muito forte & Extrema \\
\hline Madeira & & - & Forte & Muito forte \\
\hline Pau roliço & & & - & Moderada \\
\hline Lona & & & & - \\
\hline
\end{tabular}

Figura 3 - Matriz de julgamentos de diferença de atratividade para os subcritérios de Casa.

Como os subcritérios a serem avaliados já se encontram em ordem decrescente de atratividade, só é necessário preencher a matriz triangular superior (a outra parte da matriz representaria os julgamentos inversos).

A partir da construção dessa matriz, o método MACBETH é rodado e são gerados os pesos para cada um dos subcritérios. Com os julgamentos de valor expressos na Figura 3, os pesos propostos pelo MACBETH para cada subcritério de Casa são: Alvenaria $=75,25 \%$; Madeira $=20,25 \%$; Pau roliço $=4,50 \%$; Lona $=0,00 \%$. É ainda facultado ao decisor executar uma análise de sensibilidade que permite alterar os valores dos pesos, dentro dos limites estabelecidos pelos julgamentos de valor e análises de consistência. Observe-se que ao 
alterar um dos limites para um dos critérios, todos os valores de pesos para os demais critérios também são alterados, de modo a manter soma igual a 1.

Para avaliar os subcritérios de nível I, quais sejam, Casa, Poço e Energia, é aplicado o mesmo procedimento anterior. A água para o abastecimento, tanto para as necessidades dos moradores quanto dos animais, vem em primeiro plano, até mesmo antes da construção das casas de madeira ou alvenaria. Ao chegarem aos lotes, os agricultores cavavam os poços, e depois continuaram a valorizar a questão da água, com preocupação constante com a qualidade da mesma nos poços. Como a energia é muito cara, esta fica em terceiro plano, antes do poço e da construção da casa.

Com essa hierarquia, é construída uma matriz de juízos de valor, preenchida com os julgamentos de diferença de atratividade entre pares de critérios. A Figura 4 traz essa matriz preenchida, com os subcritérios ordenados em ordem decrescente de atratividade.

\begin{tabular}{|c|c|c|c|c|}
\cline { 2 - 5 } \multicolumn{1}{c|}{} & Poço & Casa & Energia & Sem instalações \\
\hline Poço & - & Forte & Muito forte & Extrema \\
\hline Casa & & - & Muito Forte & Extrema \\
\hline Energia & & & - & Forte \\
\hline SI* & & & & - \\
\hline
\end{tabular}

Figura 4 - Matriz com os julgamentos das alternativas fictícias representativas dos subcritérios de Instalações.

Com os julgamentos da Figura 4, os pesos calculados pelo MACBETH para cada subcritério de Instalações sofreram uma análise de sensibilidade por parte do decisor. Esta análise foi feita para ratificar o já exposto anteriormente, sobre a grande importância que os produtores dão para a água dos poços, até pela questão da saúde ou pela possibilidade, atual, de escassez.

A decisão do decisor foi para o seguinte conjunto de pesos: Poço $=49,98 \%$; Casa $=34,39 \%$; Energia $=15,63 \%$.

\subsection{Avaliação intercritérios}

Procedeu-se, inicialmente, à construção da matriz de preferências para estabelecer a hierarquia dos critérios. A ordenação resultante da emissão dos julgamentos do decisor sobre preferências entre pares de alternativas fictícias representantes dos critérios é a seguinte: ser proprietário do lote (LOTE); tipo de instalações (INST); usar NUAR (NUAR); percentual de dias ativos (DAT); participar de grupo não sindical (GNS); participar de associações (ASSOC); participar de cooperativas (COOP); participar de sindicatos (SIND). Ressalta-se que, segundo o decisor, para a satisfação dos produtores de Machadinho d'Oeste, participar de cooperativas ou sindicatos é indiferente (julgamento permitido pelo MACBETH).

Em seguida, foi preenchida a matriz de julgamentos de valor com os julgamentos emitidos pelo decisor sobre as diferenças de atratividade entre pares de alternativas fictícias representantes dos critérios.

De acordo com a avaliação do decisor (que, como já destacado, participou ativamente das entrevistas estruturadas no campo junto aos produtores rurais), o fator posse da terra vem em 
primeira posição, já que a partir daí eles podem tomar decisões de investimentos em infraestrutura e de produção. Em segundo lugar está a preocupação com a infra-estrutura do lote, seguida do critério relacionado a saúde, educação e lazer, pelo uso dos NUARs, já que a maioria dos produtores estão distantes da cidade. Por último, vem a forma de associativismo, sendo importante para uns e não para outros.

Com os julgamentos, o MACBETH propôs o conjunto de pesos especificados na Tabela 1. Aqui novamente, optou-se por fazer uma análise de sensibilidade, elevando o peso do critério de maior atratividade. Dar maior valor de peso para "lote" é explicado pela forte atração que os produtores têm pela posse da terra. Ser proprietário significa para eles a realização de um sonho, já que a maioria não possuía terras em seus locais de origem; eram bóia-frias, arrendatários, meeiros ou posseiros.

Tabela 1 - Limites para os pesos dos critérios que compõem o índice de bem estar social rural para os agricultores de Machadinho d'Oeste e pesos sugeridos pelo MACBETH, antes e após a análise de sensibilidade.

\begin{tabular}{ccccccc}
\hline \multirow{2}{*}{ Critério } & \multicolumn{2}{c}{ Limite inferior } & \multicolumn{2}{c}{ Limite superior } & \multicolumn{2}{c}{ Pesos sugeridos } \\
\cline { 2 - 7 } & Antes & Após & Antes & Após & Antes & Após \\
\hline LOTE & 0,2088 & 0,2094 & 0,2414 & 0,2429 & 0,2414 & 0,2421 \\
INST & 0,1865 & 0,1870 & 0,2000 & 0,2005 & 0,2000 & 0,2005 \\
NUAR & 0,1527 & 0,1531 & 0,1667 & 0,1679 & 0,1667 & 0,1671 \\
DAT & 0,1500 & 0,1504 & 0,1637 & 0,1641 & 0,1500 & 0,1504 \\
GNS & 0,1250 & 0,1244 & 0,1461 & 0,1465 & 0,1250 & 0,1253 \\
ASSOC & 0,0502 & 0,0503 & 0,0806 & 0,0808 & 0,0503 & 0,0503 \\
COOP & 0,0002 & 0,0002 & 0,0333 & 0,0333 & 0,0333 & 0,0322 \\
SIND & 0,0002 & 0,0002 & 0,0333 & 0,0333 & 0,0333 & 0,0322 \\
\hline
\end{tabular}

\subsection{Evolução do bem estar rural em Machadinho d'Oeste}

Como descrito anteriormente, o objetivo do projeto de pesquisa iniciado em Machadinho d'Oeste em 1986 é acompanhar a evolução dos sistemas de produção agrícola praticados por pequenos agricultores desta região. Ou seja, monitorar os agricultores e a agricultura da região. Com um objetivo semelhante, avaliou-se a evolução do bem estar social rural de um grupo de agricultores pesquisados pelo projeto de pesquisa.

Foi escolhida uma amostra de 73 agricultores que permaneceram em Machadinho, no mesmo lote, ao longo desses anos. Foram escolhidos como períodos de tempo para a avaliação aqueles da pesquisa de campo de 1989, 1996, 1999 e 2002 (para cada um destes anos foi rodado um modelo separado). Ou seja, ao escolher lotes que não tiveram mudança de proprietários busca-se verificar se a agricultura praticada na região promove incrementos no bem estar dos produtores rurais.

A Tabela 2 mostra as frequiências relativas de cada variável em cada ano. Nota-se que os dois critérios de maior importância relativa tiveram evolução positiva ao longo desses anos, assim como o critério referente à saúde do trabalhador $(D A T)$. Os critérios relacionados às relações sociais e de trabalho não apresentaram tendência linear de crescimento. Isso deve-se, em parte, pelo descrédito da população rural nessas instituições. 
Tabela 2 - Freqüência relativa (\%) de cada critério do índice de bem estar rural.

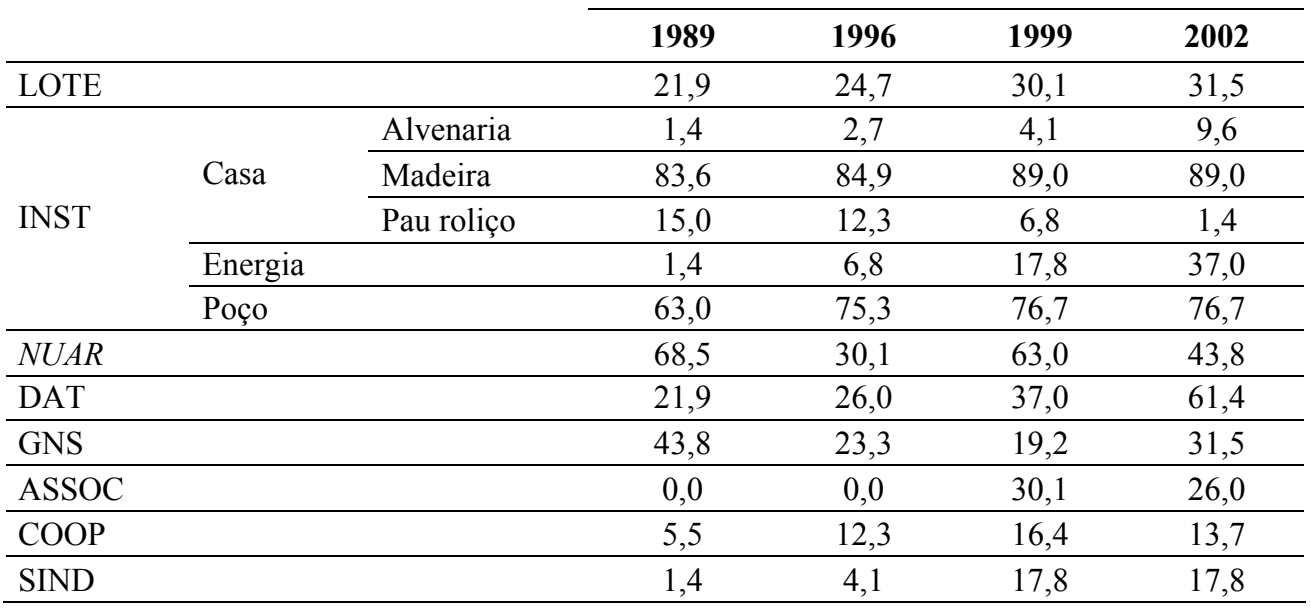

Conforme descrito, o método MACBETH sugere os pesos para os critérios e os intervalos em que podem variar, sem tornar inviáveis os quatro PPLs seqüenciais usados no método. Para calcular os pesos desconsiderou-se o peso proposto pelo MACBETH e usou-se o modelo (2) que consiste, para cada produtor, na maximização do índice de bem estar social rural, de modo que sejam respeitados os limites para os pesos (inferior e superior) sugeridos pelo MACBETH e a ordem de atratividade dos mesmos.

O problema de programação linear (2) para o cálculo dos pesos é formulado em (3) para o caso particular do problema em estudo, no qual $w_{i j}$ é a variável de decisão e representa o peso dado ao critério $j(j=L O T E, I N S T, N U A R, D A T, G N S, A S S O C, C O O P, S I N D), S_{i j}$ é o valor (normalizado) para a alternativa $i(i=1 \ldots 73)$ do critério $j$.

$\operatorname{Max}\left(\begin{array}{l}w_{i-L O T E} S_{i-L O T E}+w_{i-I N S T} S_{i-I N S T}+w_{i-N U A R} S_{i-N U A R}+w_{i-D A T} S_{i-D A T}+ \\ w_{i-G N S} S_{i-G N S}+w_{i-A S S O C} S_{i-A S S O C}+w_{i-C O O P} S_{i-C O O P}+w_{i-S I N D} S_{i-S I N D}\end{array}\right)$

sujeito a

$0,2094 \leq w_{i-L O T E} \leq 0,2429$

$0,1870 \leq w_{i-I N S T} \leq 0,2005$

$0,1531 \leq w_{i-N U A R} \leq 0,1679$

$0,1504 \leq w_{i-D A T} \leq 0,1641$

$0,1244 \leq w_{i-G N S} \leq 0,1465$

$0,0503 \leq w_{i-A S S O C} \leq 0,0808$

$0,0002 \leq w_{i-C O O P} \leq 0,0333$

$0,0002 \leq w_{i-S I N D} \leq 0,0333$

$w_{i-L O T E} \geq w_{i-I N S T} \geq w_{i-N U A R} \geq w_{i-D A T} \geq w_{i-G N S} \geq w_{i-A S S O C} \geq w_{i-C O O P}=w_{i-S I N D}$

$w_{i-L O T E}+w_{i-I N S T}+w_{i-N U A R}+w_{i-D A T}+w_{i-G N S}+w_{i-A S S O C}+w_{i-C O O P}+w_{i-S I N D}=1$

Este PPL calcula os pesos de todos os 8 critérios, com base nos intervalos obtidos no MACBETH e no postulado otimista. 
De posse dos valores dos pesos de cada critério para cada alternativa, foi calculado o índice de bem estar rural. Conforme destacado anteriormente, foram calculados pelo PPL formulado em (3) somente os pesos para os 8 critérios. Para o cálculo do índice, considerouse que os pesos dos subcritérios foram aqueles sugeridos pelo MACBETH.

A Tabela 3 resume os resultados para a abordagem otimista e traz ainda os resultados ao considerar os pesos propostos pelo MACBETH (abordagem "clássica") após a análise de sensibilidade, conforme apresentado na Tabela 1.

Segundo a abordagem otimista, verifica-se uma tendência de pequeno aumento do bem estar social ao longo dos anos, com exceção de 1996, devido aos critérios NUAR e de associativismo. Em 1996, a procura dos NUARs pelos produtores foi baixa devido à alta insatisfação destes com a capacidade de operação desses núcleos, já que naquele ano alguns postos de saúde dos NUARs passaram por dificuldades operacionais. Adicionalmente, a participação associativista é realmente problemática em Machadinho d'Oeste, por uma série de erros funcionais dessas instituições, o que causou desinteresse por parte de alguns produtores.

Globalmente, a tendência positiva indica que a atividade agropecuária na região tem sido importante, na medida em que proporciona aos seus praticantes melhoria do bem estar e de sua qualidade de vida.

Ao considerar a abordagem "clássica" nota-se comportamento semelhante ao da abordagem otimista proposta neste artigo, qual seja, uma tendência de aumento do bem estar social ao longo dos anos.

Tabela 3 - Evolução do índice médio de bem estar rural, segundo a proposta otimista de atribuição dos pesos (coluna "Otimista") e considerando os pesos sugeridos pelo MACBETH após a análise de sensibilidade (coluna "Clássica").

\begin{tabular}{lcccccccc}
\cline { 2 - 9 } & \multicolumn{2}{c}{$\mathbf{1 9 8 9}$} & \multicolumn{2}{c}{1996} & \multicolumn{2}{c}{$\mathbf{1 9 9 9}$} & \multicolumn{2}{c}{$\mathbf{2 0 0 2}$} \\
\cline { 2 - 9 } & Otimista & Clássica & Otimista & Clássica & Otimista & Clássica & Otimista & Clássica \\
\hline Média & 0,485 & 0,333 & 0,438 & 0,281 & 0,515 & 0,379 & 0,524 & 0,410 \\
Desvio padrão & 0,169 & 0,167 & 0,168 & 0,174 & 0,172 & 0,175 & 0,167 & 0,172 \\
Mínimo & 0,131 & 0,003 & 0,126 & 0,003 & 0,269 & 0,114 & 0,186 & 0,052 \\
Máximo & 0,934 & 0,718 & 0,876 & 0,797 & 0,973 & 0,828 & 0,888 & 0,830 \\
\hline
\end{tabular}

Na Figura 5 apresenta-se um gráfico com alguns resultados individualizados, segundo a abordagem otimista. Foram escolhidos para entrar nesta análise os agricultores com melhor índice em cada um dos anos. Verifica-se uma falta de homogeneidade dos resultados. O agricultor B tem uma tendência geral de melhora. O C é o que melhor exemplifica os problemas de 1996. A única tendência geral é a de uma queda significativa no bem estar logo após o ano em que cada um deles obteve o melhor índice. Tal fato pode ser decorrente de uma atitude de "acomodação", embora sejam necessários mais estudos para confirmar ou desmentir tanto o fenômeno, quanto a explicação aventada. 


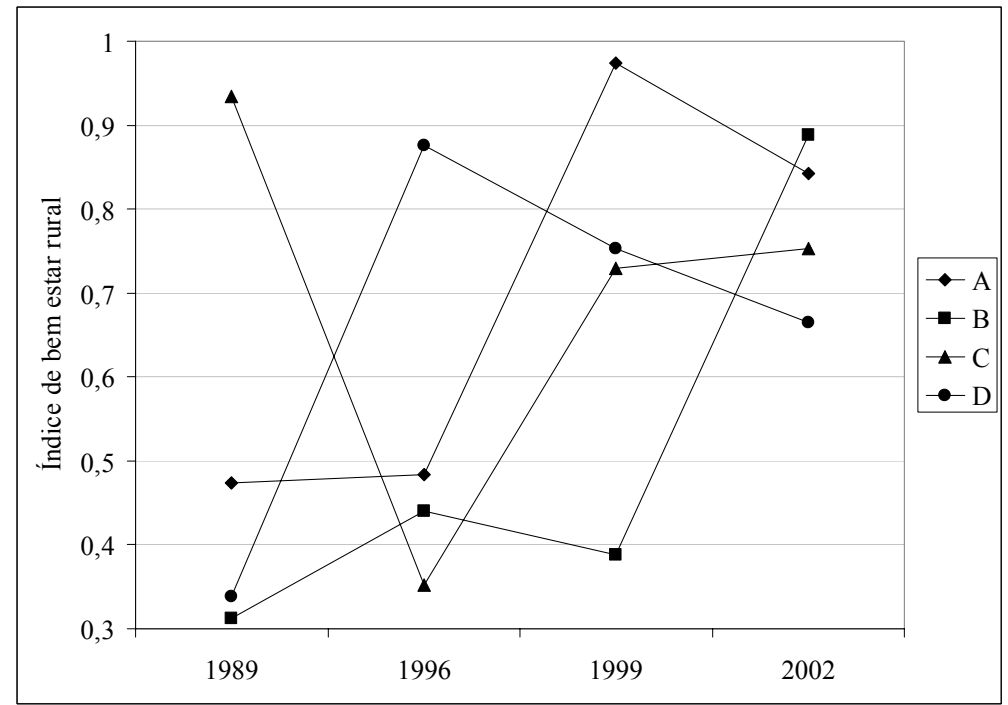

Figura 5 - Evolução do bem estar para quatro produtores.

\section{Conclusões}

A proposta de índices que avaliem bem estar, qualidade de vida e/ou satisfação social de produtores agrícolas mostra-se válida no sentido de identificar (ou não) tendências positivas da evolução da agricultura em regiões específicas. Para o caso aqui apresentado, verificou-se uma evolução positiva do índice proposto, o que indica que as práticas agrícolas da região são propulsoras de bem estar social rural.

Com o uso de um método multicritério de apoio à decisão foi possível estruturar o modelo, ponderar critérios, construir e calcular um índice de bem estar rural. Mais importante que o índice proposto é a abordagem metodológica apresentada, já que decisores diferentes podem eleger critérios diferentes, bem como ter julgamentos de valor diversos acerca dos critérios. No caso aqui apresentado, a construção do índice ficou restrita às variáveis constantes dos questionários de campo usados nos levantamentos periódicos em Machadinho d'Oeste. Mesmo assim, este índice positivo de satisfação é corroborado pelos indicadores de prosperidade e de qualidade de vida na região. A percepção de 84,4\% dos colonos em 2002 era a de que suas vidas melhoraram, e apenas $8,1 \%$ deles pensavam em sair do lote (Mangabeira et al., 2005).

A proposição da avaliação otimista permitiu que cada produtor (alternativa) escolhesse o valor do peso que maximizasse o seu índice de bem estar rural. Esta abordagem é útil no sentido de corroborar com a percepção de otimismo por parte dos agricultores.

A análise individual do total dos agricultores pode confirmar ou não a tendência de "acomodação" após alcance de um elevado nível de bem estar social. Neste sentido são necessários estudos de caráter socioestatístico para explicar o fenômeno, os quais fogem ao escopo deste artigo. Destaca-se que tendência semelhante já havia sido notada quando do estudo de medidas de eficiência para produtores desta região, nestes mesmos períodos de tempo (Gomes et al., 2006). 
Uma possibilidade interessante para análises futuras é usar como decisores um grupo de produtores rurais locais, de modo que eles elejam e julguem os critérios que expressem o que entendem como bem estar social. Destaca-se, entretanto, que a complexidade do modelo aumenta, no sentido de que esta situação é de múltiplos decisores, na qual obter consenso nem sempre é possível.

\section{Agradecimentos}

Ao CNPq, pelo apoio financeiro, por meio do Edital CNPq 19/2004 - Universal, processo n. ${ }^{\circ}$ 472838/2004-0. À Embrapa Monitoramento por Satélite, pelos dados.

\section{Referências Bibliográficas}

(1) Bana e Costa, C.A. \& Vansnick, J.C. (1995). Uma nova abordagem ao problema da construção de uma função de valor cardinal: MACBETH. Investigação Operacional, $15,15-35$

(2) Bana e Costa, C.A. \& Vansnick, J.C. (1997). Thoughts on a theoretical framework for measuring attractiveness by categorical based evaluation technique (MACBETH). In: Multicriteria Analysis [edited by Clímaco, J.], Springer-Verlag.

(3) Bana e Costa, C.A. \& Vansnick, J.C. (1999). The Macbeth approach: basic ideas, software and an application. In: Advances in Decision Analysis [edited by Meskens, N. and Roubens, M.], Kluwer Academic Publishers, Book series Mathematical Modelling: theory and applications, 4, 131-157.

(4) Bana e Costa, C.A. \& Vansnick, J.C. (2008). A critical analysis of the eigenvalue method used to derive priorities in AHP. European Journal of Operational Research, 187(3), 1422-1428.

(5) Bana e Costa, C.A.; De Corte, J.M. \& Vansnick, J.C. (2005). On the mathematical foundations of Macbeth. In: Multiple Criteria Decision Analysis: state of the art surveys [edited by J. Figueira, S. Greco and M. Ehrgott], Kluwer Academic Publishers, 409-442.

(6) Barros, R.P.; Mendonça, R.S.P. \& Duarte, R.P.N. (1997). Bem-estar, pobreza e desigualdade de renda: uma avaliação da evolução histórica e das disparidades regionais. Texto para Discussão, 454, 56p.

(7) Batistella, M. (2001). Landscape change and land-use/land-cover dynamics in Rondônia, Brazilian Amazon. Thesis (Ph.D. dissertation) - Indiana University, Bloomington, Indiana, 399p.

(8) Batistella, M.; Brondizio, E.S. \& Moran, E.F. (2000). Comparative analysis of landscape fragmentation in Rondônia, Brazilian Amazon. International Archives of Photogrammetry and Remote Sensing and Spatial Information Sciences, 33, 148-155.

(9) Batistella, M.; Robeson, S. \& Moran, E.F. (2003). Settlement design, forest fragmentation, and landscape change in Rondônia, Amazônia. Photogrammetric Engineering and Remote Sensing, 69(7), 805-812.

(10) Campos, V.R. \& Almeida, A.T. (2006). Modelo multicritério de decisão para localização de Nova Jaguaribara com VIP analysis. Pesquisa Operacional, 26(1), 91-107. 
(11) Chaves, M.C.C.; Barros, T.D.; Ramos, T.G. \& Soares de Mello, J.C.C.B. (2007). Um estudo preliminar da integração de dois métodos de apoio à decisão multicritério: VIP Analysis e MACBETH. Anais do XXXIX Simpósio Brasileiro de Pesquisa Operacional.

(12) Clímaco, J.C.N. (2003). A critical reflection on optimal decision. European Journal of Operational Research, 153(2), 506-516.

(13) Corrêa, A.M.C.J.; Crócomo, F.C.; Montebelo, M.I.L. \& Figueiredo, N.S. (2003). Bemestar, pobreza e desigualdade de rendimentos entre as pessoas ocupadas na agricultura brasileira. Pensamento \& Realidade, 12, 17-42.

(14) Corrêa, E.C. (1996). Construção de um modelo multicritério de apoio ao processo decisório. Tese (Dissertação em Engenharia de Produção), Universidade Federal de Santa Catarina.

(15) Costa, H.G.; Mansur, A.F.U.; Freitas, A.L.P. \& Carvalho, R.A. (2007). ELECTRE TRI aplicado a avaliação da satisfação de consumidores. Produção, 17(2), 230-245.

(16) Dias, L.C. \& Clímaco, J.C.N. (2000). Additive aggregation with variable interdependent parameters: The VIP analysis software. Journal of the Operational Research Society, 51(9), 1070-1082.

(17) Dyer, J.S. (1990). Remarks on the analytic hierarchy process. Journal of The Institute of Management Sciences, 36(3), 249-258.

(18) Ensslin, L.; Bana e Costa, C.A. \& Costa, A.P. (1998). Structuring the process of choosing rice varieties at the south of Brazil. In: Multicriteria Analysis for Land-Use Management [edited by Beinat, E. and Nikjamp, P.], Kluwer Academic Publishers, 1, 33-45.

(19) Ensslin, L.; Schmidt, A.M. \& Macedo, S.G. (1996). Comparação entre dois métodos de avaliação: AHP E MACBETH. Anais do XXVIII Simpósio Brasileiro de Pesquisa Operacional.

(20) Gomes, E.G.; Soares de Mello, J.C.C.B. \& Mangabeira, J.A.C. (2006). Modelos de análise de envoltória de dados na proposição de uma medida de eficiência agrícola. Anais do IX Simpósio de Pesquisa Operacional e Logística da Marinha.

(21) Gomes, L.F.A.M. (2003). Avaliações estratégicas com múltiplos critérios: porque o método AHP deve continuar a ser usado. Visão Estratégica, 1.

(22) Gomes, L.F.A.M.; Gomes, C.F.S. \& Almeida, A.T. (2002). Tomada de decisão gerencial: enfoque multicritério. Editora Atlas, 264p.

(23) Gomes, L.F.A.M.; Gonzalez-Araya, M.C. \& Carignano, C. (2004). Tomada de decisões em cenários complexos. Pioneira Thompson Learning, $168 \mathrm{p}$.

(24) Goodwin, P. \& Wright, G. (2004). Decision analysis for management judgement. $3^{\text {rd }}$ Edition. John Wiley \& Sons, 468p.

(25) Jacquet-Legreze, E. \& Siskos, Y. (1993). Assessing a set of additive utility functions for multicriteria decision making. European Journal of Operational Research, 10(2), 151-164.

(26) Kageyama, A. \& Rehder, P. (1993). O bem-estar rural no Brasil na década de oitenta. Revista de Economia e Sociologia Rural, 31(1), 23-44. 
(27) Kao, C. \& Hung, H.T. (2007). Management performance: An empirical study of the manufacturing companies in Taiwan. Omega, 35(2), 152-160.

(28) Khan, A.S.; Neiva, A.C.G.R. \& Silva, L.M.R. (2001). Projeto São José e o desenvolvimento rural no estado do Ceará. Revista de Economia e Sociologia Rural, 39(3), 143-171.

(29) Rangel, L.A.D.; Soares de Mello, J.C.C.B.; Gomes, E.G. \& Gomes, L.F.A.M. (2003). Avaliação da interiorização dos cursos da Universidade Federal Fluminense com o uso conjugado dos métodos UTA e Macbeth. Investigação Operacional, 23(1), 49-69.

(30) Madeira Júnior, A.G. (2004). Avaliação de unidades de escolta através da modelagem de apoio à decisão. Dissertação (Mestrado em Engenharia de Produção) - Universidade Federal do Rio de Janeiro.

(31) Mangabeira, J.A.C.; Miranda, E.E. de \& Gomes, E.G. (2005). Perfil agrossocioeconômico dos produtores rurais de Machadinho d'Oeste (RO), em 2002. Documentos, 38, Embrapa Monitoramento por Satélite, 114p.

(32) Mapurunga, L.F. (2000) Análise da sustentabilidade da agricultura orgânica: um estudo de caso. 2000. Dissertação (Mestrado em Desenvolvimento e Meio Ambiente) Universidade Federal do Ceará.

(33) Mariano, J.L. \& Lima, R.C. (1998). A desigualdade da renda rural no Nordeste: uma análise de desagregação do coeficiente de Gini e da sensibilidade do índice do bem estar de Sen. Análise Econômica, 16(24), 103-118.

(34) Miranda, E.E. de; Mangabeira, J.A.C.; Batistella, M. \& Dorado, A.J. (2002a). Diagnóstico agroecológico e socioeconômico dos produtos rurais de Machadinho d'Oeste (RO), em 1999. Documentos, 18, Embrapa Monitoramento por Satélite, 88p.

(35) Miranda, E.E. de; Mangabeira, J.A.C.; Gomes, E.G., Batistella, M. \& Miranda, J.R. (2002b). Café e coragem: 13 anos de colonização agrícola em Rondônia. Documentos, 17, Embrapa Monitoramento por Satélite, 102p.

(36) Miranda, E.E. de; Mangabeira, J.A.C.; Mattos, C. \& Dorado, A.J. (1997). Perfil agroecológico e socioeconômico de pequenos produtores rurais: o caso de Machadinho d'Oeste, Rondônia. Ecoforça/Embrapa-NMA, 117p.

(37) Miranda, E.E. de. (1987). Rondônia - A terra do mito e o mito da terra: os colonos do Projeto Machadinho. CNPDA, 175p.

(38) Miranda, E.E. de. (2005a). Sustentabilidade agrícola na Amazônia: 20 anos de monitoramento da agricultura em Machadinho d'Oeste-RO. Campinas: Embrapa Monitoramento por Satélite, CD-Rom.

(39) Miranda, E.E. de. (2005b). Sustentabilidade Agrícola na Amazônia - Machadinho d'Oeste. Campinas: Embrapa Monitoramento por Satélite. Disponível em: $<$ http://www.machadinho.cnpm.embrapa.br>. Acesso em: 1 nov. 2007.

(40) Roy, B. \& Bouyssou, D. (1993). Aide multicritère à la décision: méthodes et cas. Economica, 695p.

(41) Saaty, T.L. (1980). The Analytic Hierarchy Process. McGraw Hill Company, 287p. 
(42) Soares de Mello, J.C.C.B.; Gomes, E.G. \& Lins, M.P.E. (2002a). Análise multicritério da presença da Universidade Federal Fluminense com o uso do método MACBETH. Produção, 11(2), 53-67.

(43) Soares de Mello, J.C.C.B.; Gomes, E.G.; Gomes, L.F.A.M.; Biondi Neto, L. \& AnguloMeza, L. (2005). Avaliação do tamanho de aeroportos portugueses com relações multicritério de superação. Pesquisa Operacional, 25(3), 313-330.

(44) Soares de Mello, J.C.C.B.; Gomes, E.G.; Gomes, L.F.A.M.; Biondi Neto, L. \& Leta, F.R. (2003). Seleção de rota aérea com o uso do apoio multicritério à decisão. Engevista, 5(10), 71-84.

(45) Soares de Mello, J.C.C.B.; Lins, M.P.E.; Soares de Mello, M.H.C. \& Gomes, E.G. (2002b). Evaluating the performance of calculus classes using operational research tools. European Journal of Engineering Education, 27(2), 209-218.

(46) Sousa, M.C.; Khan, A.S.; Passos, A.T.B. \& Lima, P.V.P.S. (2005). Sustentabilidade da agricultura familiar em assentamentos de reforma agrária no Rio Grande do Norte. Revista Econômica do Nordeste, 26(1), 96-120.

(47) Thomaz, H.J.R. \& Gomes, L.F.A.M. (2006). Seleção de empregados na indústria de petróleo: um estudo de caso de aplicação do apoio multicritério à decisão. Anais do XXXVIII Simpósio Brasileiro de Pesquisa Operacional.

(48) $\mathrm{Yu}$, P.L. (1985). Multiple criteria decision making: concepts, techniques and extensions. Plenum Press, 402p. 